\title{
Appropriate use of tumour biomarkers for treatment with innovative drugs: A retrospective study
}

\author{
ILARIA MASSA $^{1}$, ORIANA NANNI ${ }^{1}$, MASSIMO GUIDOBONI ${ }^{2}$, GIOVANNI LUCA FRASSINETI ${ }^{3}$, \\ ANDREA ROCCA ${ }^{3}$, MARCO ANGELO BURGIO $^{3}$, LINDA VALMORRI $^{1}$, MATTIA MARRI $^{1}$, \\ ALESSANDRA PIANCASTELLI ${ }^{1}$, MARINA FAEDI $^{3}$, MAURIZIO LEONI $^{4}$, \\ STEFANO TAMBERI $^{5}$, MATTIA ALTINI ${ }^{6}$ and DINO AMADORI ${ }^{3}$
}

${ }^{1}$ Unit of Biostatistics and Clinical Trials; ${ }^{2}$ Immunotherapy Unit; ${ }^{3}$ Department of Medical Oncology, Istituto Scientifico Romagnolo per lo Studio e la Cura dei Tumori (IRST) IRCCS, Meldola 47014; ${ }^{4}$ Oncology Unit, Santa Maria delle Croci Hospital, Ravenna 48121; ${ }^{5}$ Oncology Unit, Degli Infermi Hospital, Faenza 48018; ${ }^{6}$ Healthcare Administration, Istituto Scientifico Romagnolo per lo Studio e la Cura dei Tumori (IRST) IRCCS, Meldola 47014, Italy

Received January 2, 2015; Accepted September 15, 2015

DOI: $10.3892 / 01.2015 .3967$

\begin{abstract}
Performing randomised clinical trials to address the clinical usefulness of predictive and prognostic tumour markers is a complex process for several reasons, and observational experiences may thus play an important role. The present study performed an observational retrospective analysis in Area Vasta Romagna, Italy, collecting information on tumour marker determination in 760 consecutive patients who started a new line of anticancer therapy between January and June 2010. The determination of well-known biomarkers was requested for all gastrointestinal stromal tumour (GIST) patients $(n=13)$ and for almost all breast cancer patients $(n=369)$, and targeted therapies were consequently prescribed. Conversely, Kirsten rat sarcoma viral oncogene homolog $(K R A S)$ determination in colon cancer patients $(n=177)$ was requested in $\sim 50 \%$ of advanced cases, while epidermal growth factor receptor (EGFR) determination was required in slightly more than $30 \%$ of the same patients. EGFR and KRAS determinations were requested in only $15 \%$ and $7.5 \%$ of non-small cell lung cancer (NSCLC) patients $(n=201)$, respectively. There would appear to be greater appropriateness of tumour marker determination
\end{abstract}

Correspondence to: Ms. Ilaria Massa, Unit of Biostatistics and Clinical Trials, Istituto Scientifico Romagnolo per lo Studio e la Cura dei Tumori (IRST) IRCCS, via Piero Maroncelli 40, Meldola 47014, Italy

E-mail: ilaria.massa@irst.emr.it

Abbreviations: AIFA, Italian medicines agency; AVR, area vasta Romagna; HER-2, human epidermal growth factor receptor 2; IRST IRCCS, cancer institute of Romagna; SPC, summary of product characteristics; wt, wild-type

Key words: tumour biomarker, biological drug, appropriateness, resource optimisation for breast cancer and GISTs than for colon cancer and NSCLC. Resources can be further optimised by standardising tumour marker determinations in terms of the timing of requests and the consequent use of the results for tailored treatment planning.

\section{Introduction}

In the age of personalised oncological patient care, clinical management decisions for these patients are increasingly being guided by predictive and prognostic tumour markers. As the number of available markers increases, resulting in significant use of healthcare financial resources, there is a pressing requirement for critical reviews of the body of evidence that confirms the clinical utility of these markers. Examples of these markers are oestrogen receptor and human epidermal growth factor receptor 2 (HER-2) status in breast cancer, which predict the benefit or resistance to endocrine and anti-HER-2 therapies, respectively. More recent investigations have shown that the presence of anaplastic lymphoma receptor tyrosine kinase translocations in lung cancer and the absence of Kirsten rat sarcoma viral oncogene homolog (KRAS) mutations in colorectal cancer can predict the benefit of crizotinib and anti-epidermal growth factor receptor antibodies, respectively. Considering the critical importance of prognostic and predictive tumour markers in making clinical decisions, they should be subject to the same evidence-based standards of medicine, including cost/utility analyses, as other medical interventions and practices (1).

As highlighted by Henry and Hayes (2), three steps are necessary for developing a cancer biomarker: analytic validity, clinical validity and clinical utility. Demonstrating clinical utility, which is the assessment of the effectiveness of the biomarker, in addition to its benefit-to-harm ratio, appears to be the most crucial point. In fact, clinical utility has been established for extremely few tumour markers. Conducting randomised clinical trials to address the clinical utility of these 
markers is complex in this context, being time consuming and costly, with an uncertain regulatory environment and lack of structure; therefore, observational experiences can play a significant role in evaluating and monitoring a complex framework, such as biomarker determinations and the associated drug prescription in cancer diseases.

Despite the presence of international guidelines (3-11), with their evidence-based recommendations, and national and local laws that regulate the drug prescription process, physicians are often faced with situations in daily clinical practice that can lead to biological marker determinations that are not entirely appropriate. In order to describe the current behaviour in clinical practice, the present observational retrospective study was conducted on cancer patients at different treatment stages at the Cancer Institute of Romagna (IRST, IRCCS) and in Area Vasta Romagna (AVR), a healthcare network structured into 7 local hospitals, which provides health services to a population of $\sim 1$ million people. The present study evaluated 4 selected tumour types in order to assess the types of biological marker determinations that were requested, when and how the results affected the drug prescription process and to identify any potential misuse of these determinations.

\section{Materials and methods}

Study aims. This is an observational retrospective study on consecutive cancer patients performed to evaluate the appropriateness and consistency of the biomarker determination and drug prescription processes at all hospitals in the AVR network. In particular, the study was aimed at: i) Defining which biological marker determinations are requested by oncologists in the AVR and when they are requested, for the different diseases; and ii) verifying the impact of biological marker determinations and drug prescriptions on daily clinical practice, and any potential misuse that leads to the inappropriate use of financial resources.

The study was approved by the AVR Ethics Committee and no specific written consent was required to use the patient information stored in the hospital databases.

In order to analyse the behaviour of oncologists with regard to high- and low-incidence tumours, the following 4 tumour types were selected for the study: Breast cancer, non-small cell lung cancer (NSCLC), colon cancer and gastrointestinal stromal tumours (GIST). A pool of novel target therapy drugs were selected, for which at least one biological marker was clearly recognised by the scientific community as mandatory or suggested. Major international guidelines were reviewed to identify the biological markers recommended for evaluation in each disease, and Italian and regional reference documents pertaining to treatment indications were consulted so as to identify the markers for which the determination was mandatory or suggested.

Systematic review of international guidelines. A search of guidelines that referred to tumour biomarker determinations and their application in therapy prescription was performed. A PubMed search was performed using the following keywords: 'Guideline', 'cancer biomarkers', 'biological targets', 'therapeutic choice' and 'response predictive value'. Furthermore, clinical guidelines for the selected tumour types were searched in the following databases: i) National Comprehensive Cancer Network Clinical Practice Guidelines in Oncology (3-5); ii) Scottish Intercollegiate Guidelines Network guidelines (6); American Society of Clinical Oncology guidelines (7-8); European Medicines Agency reflection paper on pharmacogenomics in oncology (April, 2008) (9); European Society of Medical Oncology (10); and the Italian Association of Medical Oncology (11).

Population. All medical records associated with all consecutive cancer patients who started a new line of antineoplastic drugs during the period between January and June 2010 were analysed. Patients of any age who were affected by one of the selected diseases at any stage and who started a systemic therapy during the considered time interval at the IRST IRCCS, and the Oncology Units of Cesena Hospital, Faenza Hospital, Lugo Hospital or Ravenna Hospital were considered. Patients enrolled in clinical trials or receiving only radiotherapy treatments or palliative care were excluded from the analysis.

A database was created that included variables on anonymous patients, on demographic data (age, gender and hospital), tumour characteristics (tumour type, date of diagnosis and tumour-node-metastasis stage at diagnosis), treatment characteristics associated with the study period (type and date of surgery, therapy setting and date of first treatment), which biological marker was requested and when, and which drug of the pool was administered during the study period and preceding it.

With regard to the evaluations of the biomarkers, these were performed according to local hospital procedures, which referred to the Society of Italian Pathology and Cytology diagnosis - Italian Division of the International Academy of Pathology guidelines (12); these are not described here as they were not the subject of this study.

Statistical analysis. All consecutive patients who met the inclusion criteria were considered and data was retrieved from each chart. Descriptive analyses were performed: Absolute frequency and relative frequency percentage were used for categorical variables, and mean, standard deviation, median, minimum and maximum were used for continuous variables. No formal hypothesis was defined. Descriptive statistics were identified for individual pathologies. The choice of a 6-month period and the considered tumours was made on the basis of the treated population, expected outcome frequencies and estimation of precision levels.

\section{Results}

Patient demographics and treatment setting. The summary results from the systematic review of the pool of drugs selected for the analysis, the associated biological markers (mandatory/suggested) and the major criteria of the summary of product characteristics (SPC) are shown in Table I (3-11). The medical records (electronic and paper) of the 760 patients treated between January 1 and June 30, 2010, were reviewed. In total, 594 out of 760 medical records were consulted electronically; paper copies were reviewed for the remaining records. According to the Oncology Unit, 340 medical records were from IRST, 128 were from the Oncology Unit of Cesena 
Table I. Drugs selected for the analysis, tumour site for which the drug is used, associated biological markers and main summary of product characteristics criteria.

\begin{tabular}{|c|c|c|c|c|}
\hline \multirow[b]{2}{*}{ Cancer type } & \multirow[b]{2}{*}{ Drug (trade name) (ref.) } & \multicolumn{2}{|c|}{ Biological marker } & \multirow[b]{2}{*}{ Main indications for prescription } \\
\hline & & Mandatory & Suggested & \\
\hline \multirow[t]{3}{*}{ Breast } & Trastuzumab (Herceptin) $(3,8,9)$ & HER-2 & - & $\begin{array}{l}\text { advBC, in association with taxanes } \\
\text { or hormone therapy; eBC/neoadjBC }\end{array}$ \\
\hline & Lapatinib (Tyverb) (9) & HER-2 & - & $\begin{array}{l}\text { advBC, in association with } \\
\text { capecitabine or AI }\end{array}$ \\
\hline & Cetuximab (Erbitux) $(4,6,13)$ & $K R A S / E G F R^{\mathrm{b}}$ & - & $\begin{array}{l}\text { advCRC, alone or in association with } \\
\text { irinotecan }\end{array}$ \\
\hline \multirow[t]{2}{*}{ Colon } & Panitumumab (Vectibix) $(4,9)$ & $E G F R, K R A S$ & - & advCRC, monotherapy \\
\hline & Bevacizumab (Avastin) & - & - & $\operatorname{advCRC}$, in association with 5-FU \\
\hline \multirow[t]{3}{*}{ NSCLC } & Gefitinib (Iressa) $(5,7,14,15)$ & $E G F R$ & - & $\operatorname{advNSCLC}^{\mathrm{a}}$ \\
\hline & Erlotinib (Tarceva) $(5,7)$ & - & $E G F R$ & $\operatorname{advNSCLC}$ \\
\hline & Bevacizumab (Avastin) & - & VEGFR & advNon-squamous NSCLC \\
\hline \multirow[t]{2}{*}{ GIST } & Imatinib (Glivec) & c-Kit & BCR-ABL & advGIST \\
\hline & Sunitinib (Sutent) & - & $\begin{array}{l}\text { PDGFR, } \\
\text { VEGFR, c-Kit }\end{array}$ & advGIST \\
\hline
\end{tabular}

${ }^{\mathrm{a} A p p r o v e d ~ i n ~ I t a l y ~ o n ~} 11$ May, 2010. ${ }^{\mathrm{b}} E G F R$ determination mandatory until July 2011. adv, advanced; BC, breast cancer; eBC, early BC; neoadjBC, neoadjuvant BC; AI, aromatase inhibitor; CRC, colorectal cancer; GIST, gastrointestinal stromal tumour; 5-FU, 5-fluorouracil; $E G F R$, epidermal growth factor receptor; KRAS, Kirsten rat sarcoma viral oncogene homolog; NSCLC, non-small cell lung cancer; HER-2, human epidermal growth factor receptor 2; PDGFR, platelet-derived growth factor; VEGFR, vascular endothelial growth factor receptor.

Table II. Patient characteristics.

\begin{tabular}{|c|c|c|c|c|}
\hline Characteristic & Breast cancer & Colon cancer & NSCLC & GIST \\
\hline Median age (range), years & $62(31-89)$ & $68(28-68)$ & $69(30-88)$ & $63(43-76)$ \\
\hline \multicolumn{5}{|l|}{ Gender, n (\%) } \\
\hline Female & $362(98.1)$ & $73(41.2)$ & $67(33.3)$ & $8(61.5)$ \\
\hline Male & $7(1.9)$ & $104(58.8)$ & $134(66.7)$ & $5(38.5)$ \\
\hline \multicolumn{5}{|l|}{ Setting n $(\%)$} \\
\hline Neoadjuvant & $16(4.3)$ & - & $5(2.5)$ & $1(7.7)$ \\
\hline Adjuvant & $182(49.3)$ & $72(40.7)$ & $10(5.0)$ & $4(30.8)$ \\
\hline \multicolumn{5}{|l|}{ Therapy line, $\mathrm{n}(\%)$} \\
\hline I line & $63(17.1)$ & $58(32.8)$ & $103(51.2)$ & $4(30.8)$ \\
\hline II line & $40(10.8)$ & $21(11.9)$ & $59(29.4)$ & $3(23.1)$ \\
\hline$>$ II line & $68(18.4)$ & $26(14.7)$ & $24(11.9)$ & $1(7.7)$ \\
\hline
\end{tabular}

NSCLC, non-small cell lung cancer; GIST, gastrointestinal stromal tumour.

Hospital, 66 from Faenza Hospital, 88 from Lugo Hospital and 138 from Ravenna Hospital. A total of 369 medical records were from breast cancer patients, 177 were from colon cancer patients, 201 were from NSCLC patients and 13 were from GIST patients (Table II).

In total, $49.3 \%$ of the patients treated for breast cancer received therapy in the adjuvant setting and $4.3 \%$ received therapy in the neoadjuvant setting, whereas the remaining patients were divided fairly evenly between first (17.1\%) and second $(10.8 \%)$ lines of therapy and beyond (18.4\%).
For colon cancer, $40.7 \%$ of patients were in the adjuvant setting, $32.8 \%$ were in the first-line setting, $11.9 \%$ were in the second-line and $\sim 14.7 \%$ were in higher than the second-line setting; more than half of the patients exhibited advanced disease.

For NSCLC, the majority of patients were in the first-line setting (51.2\%), with $29.4 \%$ in the second-line and only $11.9 \%$ in higher than the second-line setting. Only a few patients were receiving therapy in the neoadjuvant $(2.5 \%)$ or adjuvant $(5.0 \%)$ setting. 
Table III. HER-2-positive breast cancer patients and HER-2-positive patients treated with trastuzumab (prior to or during the study period).

\begin{tabular}{|c|c|c|c|c|c|}
\hline \multirow[b]{2}{*}{ Parameter } & \multirow{2}{*}{$\begin{array}{c}\text { All HER-2 } \\
\text { evaluated patients }\end{array}$} & \multicolumn{2}{|c|}{$\begin{array}{l}\text { HER-2-positive } \\
\text { patients }\end{array}$} & \multicolumn{2}{|c|}{$\begin{array}{l}\text { HER-2-positive patients } \\
\text { treated with trastuzumab }\end{array}$} \\
\hline & & $\mathrm{n}$ & $\%$ & $\mathrm{n}$ & $\%$ \\
\hline \multicolumn{6}{|l|}{ Setting } \\
\hline Neoadjuvant & 16 & 4 & 25.0 & 4 & 100.0 \\
\hline Adjuvant & 191 & 62 & 32.4 & 59 & 95.2 \\
\hline \multicolumn{6}{|l|}{ Therapy line } \\
\hline I line & 56 & 18 & 32.1 & 17 & 94.4 \\
\hline II line & 34 & 11 & 32.4 & 11 & 100.0 \\
\hline$>$ II line & 66 & 13 & 19.7 & 12 & 92.3 \\
\hline Total & 363 & 108 & 29.8 & 103 & 95.4 \\
\hline
\end{tabular}

HER-2, human epidermal growth factor receptor 2.

A total of 13 GIST patients were also evaluated, with 7.7 and $30.8 \%$ in the neoadjuvant and adjuvant settings, respectively, and $30.8,23.1$ and $7.7 \%$ in the first-line, second-line and higher than second-line settings, respectively.

Breast cancer. In addition to the other routine biological markers currently used for the characterisation of breast cancer, HER-2 determination was performed in 363 (98.4\%) of the 369 patients evaluated. HER-2 determination was requested immediately after diagnosis $(75.9 \%)$ or prior to the start of therapy (22.5\%), but never after the beginning of chemotherapy. HER-2 marker determination data were missing for $6(1.6 \%)$ patients in the group, mainly due to the fact that for 4 of the 6 patients, treatment with trastuzumab was excluded due to an advanced age ( $>80$ years), and for the remaining 2 patients, it was excluded as surgery had been performed $>20$ years previously; therefore, it was impossible to obtain tissue tumour blocks in order to perform the tests. In total, 108 patients out of 363 were HER-2-positive (29.8\%); 88 of these received trastuzumab in the referral period, while 15 patients had already received trastuzumab prior to the study period, meaning that a total of 103 out of 108 (95.4\%) HER-2-positive patients received trastuzumab. The remaining 5 HER-2-positive patients had never been treated with trastuzumab (Table III). The reasons for this result were investigated in-depth in the clinical diary: 1 patient (64 years old, adjuvant setting) refused therapy; 2 patients ( $>80$ years old, adjuvant setting) were considered ineligible for trastuzumab treatment in view of their age; 1 patient (45 years old, first-line setting) was found to be HER-2-positive at the time of diagnosis (2003) at IHC (result, 3+; HercepTest; Dako, Glostrup, Denmark), but was found to be negative on fluorescence in-situ hybridisation (FISH) in October 2010; and 1 patient (78 years old, higher than second-line setting) did not receive trastuzumab due to cardiac comorbidities.

With regard to lapatinib, 12 patients were treated with this drug; 8 of these received the drug in the study period and all of these patients had previously received trastuzumab. Only
4 patients were treated with bevacizumab in the first-line setting, and 2 of these received the drug in the referral period.

Colon cancer. In the adjuvant setting, no determination was requested. In the advanced setting (105 patients), KRAS and epidermal growth factor receptor $(E G F R)$ marker determinations were performed in $50(47.6 \%)$ and $35(33.3 \%)$ patients, respectively. KRAS and EGFR marker determinations were available for 30 out of the 105 patients treated for the advanced disease $(28.6 \%)$.

In the 50 patients with $K R A S$ determination, 37 showed a KRAS wild-type (wt) status, whereas in the remaining 13, mutated KRAS was found.

With regard to the EGFR status, for 16 patients the gene was found to be amplified, while in the remaining 19 it was not. A total of 26 colon cancer patients were treated with cetuximab; 17 of these patients received the drug during the analysis period, consisting of 4 patients as first-line treatment, 4 patients as second-line treatment and 9 patients as a higher than second-line treatment. All patients treated with cetuximab carried the wt KRAS, but EGFR determination, requested by SPC at that time (therefore, mandatory for administration), was performed in only 14 cases, 10 of which showed amplified $E G F R$. Therefore, in 4 cases, cetuximab was administered in the absence of EGFR amplification, whereas in 10 cases, $E G F R$ determination was not requested, in contrast with what is prescribed by the SPC (Table IV). Among advanced cancer patients who did not receive cetuximab, 13 had wt KRAS, but for 8 patients $E G F R$ was not amplified; therefore, cetuximab had not been administered correctly, in accordance with the SPC. The remaining 5 wt KRAS patients did not receive cetuximab in the study period and received chemotherapy treatment such as 5-fluorouracil, irinotecan, oxaliplatin and bevacizumab according to the SPC. Of the 42 colon cancer patients treated with bevacizumab, 17 received the drug during the referral period. Although panitumumab was never prescribed during the study period, 3 colon cancer patients in the late stage setting (higher than second-line setting) had 
Table IV. Treatment with cetuximab according to EGFR status in advanced wild-type $K R A S$ patients $(\mathrm{n}=37)$.

\begin{tabular}{lccc}
\hline & \multicolumn{2}{c}{ Cetuximab } & \\
\cline { 2 - 3 } EGFR status & Administered & Not administered & Total \\
\hline Amplified & 10 & 1 & 11 \\
Not amplified & 4 & 8 & 12 \\
Not determined & 10 & 4 & 14 \\
Total & 24 & 13 & 37 \\
\hline
\end{tabular}

$E G F R$, epidermal growth factor receptor; KRAS, Kirsten rat sarcoma viral oncogene homolog.

been treated with the drug in previous treatments, but it had been prescribed in the context of an expanded access program.

NSCLC. With regard to histological types, the study population was comprised of $75.6 \%$ adenocarcinoma and $21.9 \%$ squamocellular carcinoma cases, whereas in the remaining $2.5 \%$ of the population, the histological diagnosis was missing due to a lack of tumour tissue; this is quite a common situation in lung cancer patients and only allows for cytological diagnosis. EGFR marker determination was requested for 30 out of the 201 patients of the group, resulting in 23 wt EGFR and 7 mutated EGFR cases. With regard to the timing of the request, 13 patients were in the first-line setting, 11 in the second-line setting and 6 in higher than the second line. With regard to the association between histological type and EGFR request, for $22(73.3 \%)$ out of the 30 requested $E G F R$ determinations the histological type was adenocarcinoma, while in 3 cases (10.0\%) the histology was squamocellular carcinoma and for the remaining 5 cases (16.6\%) the histological type was unknown. KRAS marker determination was requested less frequently $(\mathrm{n}=15)$ and always together with $E G F R$ assessment. Of the 83 second-line/higher than second-line NSCLC patients, $41(49.4 \%)$ were treated with erlotinib during the analysis period. EGFR marker determination was requested for only 8 patients treated with erlotinib.

None of the patients received gefitinib (gefinitib authorisation for Italy was only provided on 11 May 2010) or bevacizumab during the study period.

GIST. c-Kit marker determination was requested for all patients included in the analysis. Marker determination data were found in the medical records of only 2 patients of the PDGFR group. All patients were treated with imatinib during the analysis period or in previous therapies, and 3 out of 13 patients were also treated with sunitinib.

\section{Discussion}

The present survey, performed in 5 Oncology Units of hospitals in AVR, showed that research results are promptly translated into daily practice. A substantial appropriateness of biological determination requests, a correct use of these data for the therapeutic choice and an overall consistency between different oncologists was found. Whilst these results were particularly robust for breast cancer and GIST patients, the results for colon and lung cancer could be slightly improved.

The population included in the study, due to its consecutive nature and the fact that the AVR clinical network provides healthcare services to $>96 \%$ of the resident population, could be considered representative of all cancer patients resident in the AVR affected by breast cancer (30\% of all female cancer patients), NSCLC (12\% of all cancer patients), colorectal cancer (10\%) and GISTs (0.8\%) (data taken from the Romagna Tumour Registry).

A high level of homogeneity in the management of breast cancer was observed among oncologists from the various hospitals of the AVR network. HER-2 determinations were, in fact, requested at diagnosis in almost all patients included in the study and represented the key driver for the therapeutic choice. In accordance with the standard of treatment at that time, trastuzumab was prescribed, if indicated, early on in breast cancer treatment to maximise its potential therapeutic benefit. Few HER-2-positive patients had not been treated with trastuzumab and these rare cases were mainly due to age and comorbidity. Strong drug prescription compliance was noted and all patients treated with trastuzumab were found to be HER-2-positive.

Trastuzumab was an example of the high efficacy of target therapy; the biological determination was then promptly introduced into clinical practice.

With respect to colon cancer, EGFR and KRAS marker determinations were mandatory in order to treat patients with cetuximab but, in actual fact, KRAS was requested in $\sim 50 \%$ of colon cancer patients in the advanced stage, while EGFR determination was requested for only a little more than $30 \%$ of the same patients. At the time of the survey, the hypothesis that $E G F R$ was not predictive of response to cetuximab was widespread amongst oncologists due to data from retrospective studies $(16,17)$, however, the Italian Medicines Agency (AIFA) did not modify the cetuximab indication, removing the obligatory EGFR determination, until July 2011. The misalignment between what was already accepted by the scientific community and what was requested by AIFA led to an increased use of financial resources in terms of the evaluation of EGFR status and the inappropriateness of cetuximab prescription, as certain patients with wt $K R A S$ did not receive cetuximab as they did not have amplified $E G F R$. No EGFR or $K R A S$ determinations were requested in the adjuvant setting, which is appropriate, as they have a predictive, but not a prognostic value (4). Only a small percentage (10\%) of patients who were candidates to receive cetuximab did not receive it during the study period. In all probability, a number of these patients will receive the target drug at a later date and others, in view of age, performance status or population comorbidity, will never receive cetuximab; therefore, KRAS mutation data will remain unused. In 2009, a commission for drug evaluation with the task of defining evidence-based recommendations commenced its activity in the Emilia Romagna region. This commission refers to the Grading of Recommendations Assessment, Development and Evaluation method (18) to formulate its recommendations and performs periodic audits at hospitals to evaluate prescription appropriateness. In this regional context, considering the role of other biomarkers, such as $B R A F$ and all $R A S$ mutations, which have an impact not only on therapeutic 
strategies, but also on the use of financial resources, the global management of the advanced colon cancer patient in terms of chemo-biological treatment, and choice and timing of biological determinations remains a crucial issue in daily clinical practice.

With regard to NSCLC, for certain target drugs, the context was more uncertain at the time of this retrospective survey than it is now, and the use of one of the key target drugs, gefinitib, had still not been authorised in Italy. Although EGFR determination was requested in $\sim 15 \%$ of patients, the results were not taken into consideration for the choice of therapy. For patients treated with erlotinib, EGFR determination was not mandatory, but was performed in $20 \%$ of cases, thus making it of questionable value. The majority of $E G F R$ determinations were requested for the adenocarcinoma histotype in accordance with indications in the literature (5). Overall, it was observed that $E G F R$ determination was requested at different stages of NSCLC history, confirming that, at the time of this monitoring survey, different approaches to NSCLC were possible. Nearly two-thirds of all patients with lung adenocarcinoma harbour at least one mutation in known lung cancer driver genes, and patients whose treatment is targeted specifically to those drivers show improved survival (19). Thus, close monitoring of biological determinations, their timing and the subsequent choice of treatment may be required to optimise therapeutic appropriateness and cost-effectiveness.

c-Kit determination undeniably plays a crucial role in the treatment of GISTs. Indeed, for all patients included in the present analysis, this determination was requested at the diagnosis stage. On the other hand, although several guidelines include PDGFR among the relevant biological markers that should be investigated for the treatment of GISTs, this marker does not appear to be in use. The GIST model is a paradigmatic example of personalised medicine, where biomarker determination is crucial for diagnosis and therapy, since there is a strong correlation between biomarker and target therapy. Achieving this type of 'personalised medicine' for all other cancer diseases is desirable.

In order to achieve this objective, it may be effective to implement an organisational model, such as the network between the Oncology units existing in AVR, which includes the centralisation of certain specific high-cost activities and the establishment of tumour-specific groups of physicians who can provide a standardised and appropriate approach to tumour care. In such a context, the prompt translation of research results into clinical practice and permanent monitoring of the appropriate use of resources, in terms of pharmaceutical expenditure and biomarker determinations, is ongoing.

Nonetheless, in the era of personalised medicine, an increasing number of novel biological markers are being identified, that could be used in practice, but for which clinical utility demonstrated with randomised controlled trials is often absent. Moreover, their clinical value in the real world of clinical practice is unknown; therefore, an important contribution to clarify the role of these determinations can be offered by retrospective studies and/or monitoring surveys such as in the present study. The methodological limitations of this type of study are well known, particularly in terms of missing data and selection bias, but in the present survey these risks were reduced since electronic chart records are properly registered and a priori patient selection was not performed. Another limitation can be due to the fact that therapeutic indications for each target drug, as outlines in the SPC, constantly change over time and it can occasionally be difficult to trace the correct indication that was mandatory at a specific time. It would be desirable for the role of retrospective experiences to be increasingly recognised in order to identify what is happening in the real world of cancer patients, not only for biological marker determinations, but also for other complex aspects of cancer patient management.

\section{Acknowledgements}

The authors wish to thank Ursula Elbling (IRST IRCCS) for editing the original manuscript.

\section{References}

1. McShane LM and Hayes DF: Publication of tumor marker research results: The necessity for complete and transparent reporting. J Clin Oncol 30: 4223-4232, 2012.

2. Henry NL and Hayes DF: Cancer biomarkers. Mol Oncol 6: 140-146, 2012.

3. NCCN: Clinical Practice Guidelines in Oncology. Breast Cancer Screening and Diagnosis, V.1.2010. http://www.nccn.org/professionals/physician_gls/f_guidelines.asp. Accessed November 15, 2014.

4. NCCN: Clinical Practice Guidelines in Oncology. Colon cancer, V.I.2010. http://www.nccn.org/professionals/physician_gls/f_ guidelines.asp. Accessed November 15, 2014.

5. NCCN: Clinical Practice Guidelines in Oncology. Non small lung cell cancer, V.2.2010. http://www.nccn.org/professionals/physician_ gls/f_guidelines.asp. Accessed November 15, 2014.

6. Scottish Intercollegiate Guidelines Network: SIGN Guidelines. http://www.sign.ac.uk/guidelines/published/. Accessed November 15, 2014.

7. Azzoli CG, Temin S, Aliff T, et al: 2011 Focused update of 2009 American society of clinical oncology clinical practice guideline update on chemotherapy for stage IV non-small-cell lung cancer. J Clin Oncol 29: 3825-3831, 2011.

8. Harris L, Fritsche H, Mennel R, et al: American society of clinical oncology 2007 update of recommendations for the use of tumor markers in breast cancer. J Clin Oncol 25: 5287-5312, 2007.

9. EMEA: Reflection paper on pharmacogenomics in oncology-draft guideline. http://www.ema.europa.eu/docs/en_GB/document_ library/Scientific_guideline/2009/09/WC500003866.pdf. Accessed November 15, 2014.

10. ESMO: Clinical Practice Guidelines. http://www.esmo. org/Guidelines. Accessed November 15, 2014.

11. AIOM: Clinical Guidelines. http://www.aiom.it/. Accessed November 15, 2014.

12. SIAPEC-IAP: Guidelines. www.siapec.it. Accessed November 15, 2014

13. Karapetis CS, Khambata-Ford S, Jonker DJ, et al: K-ras mutations and benefit from cetuximab in advanced colorectal cancer. $\mathrm{N}$ Engl J Med 359: 1757-1765, 2008.

14. Cohen M, Williams GA, Sridhara R, et al: United States food and drug administration approval summary: Gefitinib (ZD1839; Iressa) tablets. Clin Cancer Res 10: 1212-1218, 2004.

15. Lynch TJ, Bell DW, Sordella R, et al: Activating mutations in the epidermal growth factor receptor underlying responsiveness of non-small-cell lung cancer to gefitinib. New Engl J Med 350: 2129-2139, 2004.

16. Scartozzi M, Bearzi I, Berardi R, et al: Epidermal growth factor receptor (EGFR) status in primary colorectal tumors does not correlate with EGFR expression in related metastatic sites: Implications for treatment with EGFR-targeted monoclonal antibodies. J Clin Oncol 22: 4772-4778, 2004.

17. de Castro-Carpeño J, Belda-Iniesta C, Casado Sáenz E, et al: EGFR and colon cancer: A clinical view. Clin Transl Oncol 10: 6-13, 2008.

18. Atkins D, Best D, Briss PA, et al: Grading quality of evidence and strength of recommendations. BMJ 328: 1490, 2004.

19. Patel JD, Krilov L, Adams S, et al: Clinical cancer advances 2013 : Annual report on progress against cancer from the American society of clinical oncology. J Clin Oncol 32: 129-160, 2014. 